\title{
INCORPORACIÓN DE ENTORNOS TECNOLÓGICOS DE APRENDIZAJE A LA CULTURA ESCOLAR: PROYECTO DE INNOVACIÓN EDUCATIVA EN MATEMÁTICAS Y CIENCIAS EN ESCUELAS SECUNDARIAS PÚBLICAS DE MÉXICO
}

\section{Teresa Rojano (*)}

SÍNTESIS: En este artículo se describe la experiencia de un proyecto de innovación educativa desarrollado en México por iniciativa de la Secretaría de Educación Pública y el Instituto Latinoamericano de la Comunicación Educativa, en el que se incorpora el uso de las tecnologías de la información y la comunicación a la enseñanza de las matemáticas y las ciencias en la escuela secundaria pública. Las características de este proyecto en cuanto a los principios básicos de su diseño, a la metodología de su implementación, a los grupos participantes provenientes de distintas comunidades (académicos, desarrolladores de software especializado, autoridades educativas, maestros y alumnos), a su trasfondo internacional, y a su estrecha relación con una rigurosa investigación educativa, hacen que los resultados que reporta, después de una experiencia de cinco años, puedan tener relevancia en ámbitos que rebasan su contexto local de prueba. Los resultados obtenidos de la puesta en marcha del proyecto en cuestión, Enseñanza de la Física y las Matemáticas con Tecnología (EFIT-EMAT), han influido en la elaboración de las secciones de informática educativa del Plan Nacional de Educación 2001-2006, y en la formulación de las actuales propuestas de reforma curricular para las materias de matemáticas y ciencias de la enseñanza secundaria en México. Algunos resultados parciales han servido de referencia para la incorporación explícita al currículo de otros países del uso de entornos tecnológicos de aprendizaje, como el de la hoja electrónica de cálculo para la resolución de problemas aritméticoalgebraicos y para la modelación matemática en ciencias.

(*) Asesora de la Subsecretaría de Educación Básica y Normal de la Secretaría de Educación Pública en México (SEP); para este trabajo contó con la colaboración de Elvia Perrusquía M., de la Dirección General de Materiales y Métodos Educativos de la SEP. 


\begin{abstract}
SÍNTESE: Neste artigo se descreve a experiência de um projeto de inovação educacional desenvolvido no México por iniciativa da Secretaria de Educação Pública e do Instituto Latino-americano da Comunicação Educacional, no qual se incorpora o uso das tecnologias da informação e da comunicação ao ensino das matemáticas e das ciências no ensino médio da escola pública. As características deste projeto quanto aos princípios básicos de seu desenho, à metodologia de sua implementação, aos grupos participantes provenientes de distintas comunidades (acadêmicos, desenvolvedores de software especializado, autoridades educacionais, professores e alunos), a seu cenário internacional, e a sua estreita relação com uma rigorosa investigação educacional, fazem que os resultados a que remete, depois de uma experiência de cinco anos, possam ter relevância em âmbitos que ultrapassam seu contexto local de prova. Os resultados obtidos da execução do projeto em questão, Ensino da Física e das Matemáticas com Tecnologia (EFIT-EMAT), têm influenciado na elaboração das seções de informática educacional do Plano Nacional de Educação 2001-2006, e na formulação das atuais propostas de reforma curricular para as matérias de matemática e ciências do ensino médio no México. Alguns resultados parciais têm servido de referência para a incorporação explícita ao currículo de outros países do uso de entornos tecnológicos de aprendizagem, como o da página eletrônica de cálculo para a resolução de problemas aritmético-algebraicos e para a modelação matemática em ciências.
\end{abstract}

\title{
1. INTRODUCCIÓN
}

El uso de las tecnologías de la información y la comunicación (TIC) en la escuela tiene ya una historia de más de 20 años, si consideramos los intentos y los experimentos llevados a cabo en países pioneros en este campo. Sin embargo, la incorporación sistemática y oficial de tales herramientas a los sistemas escolares ha sido mucho más reciente, y aún más recientes han sido los estudios y evaluaciones que dan cuenta de los resultados de dicha incorporación. En general, los resultados más relevantes reportados en distintas latitudes coinciden en que los alumnos experimentan un aprendizaje significativo a través de un uso apropiado de las TIC (Dunham y Dick, 1994; Boers-van Oosterum, 1990; Rojano, 1996); que los maestros con poca experiencia en el uso de las TIC tienen gran dificultad en apreciar su poder como herramientas de aprendizaje, y, como consecuencia de lo anterior, que de no atenderse la carencia de conocimiento tecnológico de los docentes, las TIC no tendrán una influencia importante en la cultura del aula (McFarlane, 2001). A su vez, estos resultados han conducido a instituciones educativas y a instancias 
políticas de diversos países a definir su posición respecto a distintas concepciones del uso de tales tecnologías en educación.

En la actualidad se reconocen internacionalmente tres concepciones bien diferenciadas: las TIC como un conjunto de habilidades 0 competencias; las TIC como un conjunto de herramientas o de medios de hacer lo mismo de siempre pero de un modo más eficiente; las TIC como un agente de cambio con impacto revolucionario (McFarlane et al., 2000). La primera propone a las TIC como materia de enseñanza, lo cual conduce a logros en el nivel de las competencias informáticas mismas; sin embargo, esto no garantiza que dichos logros se reflejen automáticamente en otras áreas curriculares (por ejemplo, las matemáticas o las ciencias naturales).

En la segunda se pone énfasis en la relación de las TIC con el currículo, y consiste en agregar elementos de tecnología informática a las tareas de aprendizaje para un mejor logro de los objetivos planteados por el currículo vigente. Si bien bajo esa perspectiva se está en posibilidad de alcanzar con más eficiencia dichos objetivos, una de las mayores debilidades de tal enfoque reside en que los modelos que de él surgen tienden a medir los resultados de su aplicación, del mismo modo en que se miden los resultados de realizar las tareas sin el uso de las TIC. En otras palabras, esos modelos anticipan el efecto de las TIC en el logro de objetivos, tal y como lo prevén los sistemas de evaluación estandarizados. Esto último ha sido muy cuestionado por los especialistas en aprendizaje mediado por las TIC, que se basan en teorías del aprendizaje situado (Lave, 1988; Rogoff y Lave, 1984; Wertsch, 1991), y cuyas consideraciones conducen a concluir que el aprendizaje que se lleva a cabo en un entorno tecnológico no siempre se transfiere de manera espontánea a otro tipo de entornos (por ejemplo, el de papel y lápiz), de modo que, aunque existen coincidencias en una variedad de estudios en los que este uso de las TIC promueve el trabajo colectivo y mejora la capacidad de los alumnos para plantear preguntas y tomar decisiones apropiadas, sus logros no se ven reflejados en las calificaciones finales de los estudiantes. De ahí que los intentos de balance del impacto de las TIC sobre los objetivos educativos ha sido, en términos generales y en el mejor de los casos, más o menos favorable. Cabe señalar, además, que la segunda concepción ha recibido severas críticas por el hecho de centrarse en el estudiante como usuario de la tecnología, sin dar la debida importancia al papel del maestro. 
Finalmente, la tercera concepción, que considera a las TIC como agentes de cambio y con una gran potencialidad de revolucionar las prácticas en el aula, está hoy muy difundida en los medios académicos (comunidad de especialistas y de investigadores del uso de las TIC en educación; véase por ejemplo Crook, 1994); sin embargo, es difícil encontrar ejemplos de su implementación en los sistemas educativos. Este acercamiento que posibilita reformular a fondo lo que hay que enseñar, cómo enseñarlo y el rol del profesor, ha entrado en conflicto en algunos países con la cultura escolar existente, generada en buena medida por un currículo conservador, que no da espacio a un alumno que ha adquirido cierta autonomía en el aprendizaje a través de un uso intensivo de las TIC fuera de la escuela (Facer et al., 2000). Esta situación es propia de los países en los que el acceso de la sociedad a las TIC, directo y generalizado, ha tenido lugar en el hogar antes que en la escuela. Este no es el caso de países en desarrollo, como México, en donde los alumnos, en su mayoría, tendrán su primer acercamiento al uso directo de las TIC en el ámbito escolar.

Lo anterior representa un doble reto para los sistemas educativos en los países en desarrollo, pues además de incorporar las TIC a la escuela a través de un uso apropiado para la enseñanza y el aprendizaje, se debe afrontar el hecho de que la mayor parte de los docentes y de los alumnos no posee las competencias informáticas básicas. Concebir modelos de incorporación de las TIC con la tercera concepción implica, junto a ese doble reto, lidiar con las prácticas generadas por un currículo conservador, tal como ya se señaló.

A pesar de las dificultades que pueden anticiparse con la aplicación de la tercera concepción, es precisamente la condición de agentes de transformación la que dicha concepción asigna a las TIC, Io que las hace dignas de tomarse en cuenta en el momento de concebir intervenciones deliberadas para cambiar en lo esencial los modelos pedagógicos, las prácticas en el aula y los contenidos curriculares en sistemas educativos en los que se ha planteado la necesidad de emprender reformas de esta naturaleza, a fin de conducir a los estudiantes hacia un aprendizaje significativo y satisfactorio.

En este artículo se estudia de manera sucinta la experiencia de un proyecto de innovación educativa desarrollado en México, en el que se incorporó el uso de las TIC a la enseñanza de las matemáticas y las ciencias en la escuela secundaria desde la perspectiva de la tercera concepción. El principal propósito de este proyecto fue poner a prueba modelos de uso 
de las TIC en los que, a la vez que se incidiera en el mejoramiento del aprendizaje de temas curriculares clásicos, se tuviese una influencia en la transformación de las prácticas en el aula, y se incursionara en la enseñanza de nuevos contenidos que permitieran al alumno el acceso a ideas importantes en matemáticas y ciencias mediante el trabajo en entornos tecnológicos. Los propios componentes del propósito del proyecto explican su ubicación en la tercera concepción y no en otra, pero a medida que se avance en la lectura de este documento se hará más clara la toma de posición que sirvió de premisa para su diseño, implementación, evaluación y seguimiento.

Los resultados obtenidos de la puesta en marcha del proyecto Enseñanza de la Física y las Matemáticas con Tecnología (EFIT-EMAT), sirven hoy de referencia para el programa de reforma educativa en puertas en el sistema de educación secundaria en México.

Si bien se trata de modelos que en su fase piloto muestran bondades y limitaciones en su contexto local de prueba, tanto los fundamentos que permiten concebirlos como la metodología de investigación aplicada que los valida, pueden resultar relevantes para sistemas educativos con motivaciones similares acerca del uso de entornos tecnológicos de aprendizaje en la educación básica.

\section{ENSEÑANZA DE LAS MATEMÁTICAS Y LA FÍSICA CON TECNOLOGÍA: PRINCIPIOS BÁSICOS DE DOS MODELOS DE USO DE LAS TIC EN EL AULA}

En 1997 la Subsecretaría de Educación Básica y Normal de la Secretaría de Educación Pública (SEP), en colaboración con el Instituto Latinoamericano de la Comunicación Educativa (ILCE), tomó la iniciativa de poner en marcha el proyecto de innovación y desarrollo educativo Enseñanza de la Física y las Matemáticas con Tecnología (EFIT-EMAT), con los siguientes objetivos generales:

- Incorporar sistem ática y graduam ente eluso de las TIC en la escuela secundaria pública para la enseñanza de las Matemáticas y de la Física. 
- Poner en práctica el uso significativo de las TIC basándose en un modelo pedagógico orientado a mejorar y a enriquecer el aprendizaje de los contenidos curriculares.

- Explorar el uso de las TIC para la enseñanza de contenidos más allá del currículo, con base en el acceso a ideas importantes en ciencias y matemáticas.

Elproyecto EFIT-EMAT, que se traduce en modelos específicos para la enseñanza de la Física (EFIT) y las Matemáticas (EMAT) con tecnología, se concibe bajo los siguientes principios:

- Didáctico, mediante el cual se diseñan actividades para el aula siguiendo un tratamiento fenomenológico ${ }^{1}$ de los conceptos que se enseñan.

- De especialización, por el que se seleccionan herramientas y piezas de software de contenido. Los criterios de selección se derivan de didácticas específicas acordes con cada materia (Física y Matemáticas).

- Cognitivo, por cuyo conducto se seleccionan herramientas que permiten la manipulación directa de objetos matemáticos y de modelos de fenómenos mediante representaciones ejecutables.

- Empírico, bajo el cual se seleccionan herramientas que han sido probadas en algún sistema educativo.

- Pedagógico, por cuyo intermedio se diseñan las actividades de uso de las TIC para que promuevan el aprendizaje colaborativo ${ }^{2}$ y la interacción entre los alumnos, así como entre profesores y alumnos.

1 Tratamiento fenomenológico: la idea central es que los conceptos son organizadores de fenómenos; así, la contextualización no es una mera ambientación de las actividades de enseñanza, sino que las situaciones que se utilizan corresponden a comportamientos de fenómenos que, en cierto modo, forman parte de la esencia del concepto que se busca enseñar; es decir, son parte de la fenomenología del concepto.

${ }^{2}$ Este modelo consiste en la distribución de tareas a diferentes equipos de alumnos dentro del grupo, los cuales intercambian los resultados de su trabajo en sesiones plenarias de discusión conducidas por el profesor. En el caso de EFIT cada equipo trabaja con una pieza de tecnología diferente, y en EMAT todos los equipos trabajan con el mismo software y con la misma actividad. 
- De equidad, con el que se seleccionan herramientas que permiten a los alumnos de secundaria el acceso temprano a ideas importantes en ciencias y matemáticas.

Del conjunto de toma de decisiones para el diseño de los modelos, una de las más complejas era la de selección de herramientas. Estos principios permitieron formular criterios de selección, los cuales indican que las herramientas debieran:

- Estar relacionadas con un área específica de la matemática o de la física escolar.

- Contar con representaciones ejecutables de objetos, conceptos y fenómenos de la matemática y de la ciencia.

- Permitir un tratamiento fenomenológico de los conceptos matemáticos y científicos.

- Ser útiles para abordar situaciones que no pueden estudiarse con los medios tradicionales de enseñanza.

- Poder utilizarse con base en el diseño de actividades que promuevan un acercamiento social del aprendizaje.

- Permitir promover prácticas en el aula en las que el profesor guía el intercambio de ideas y las discusiones grupales, al tiempo que actúa como mediador ${ }^{3}$ entre el estudiante y la herramienta.

La identificación de un conjunto de entornos tecnológicos de aprendizaje que cumplieran con estos criterios hizo posible el diseño de los modelos pedagógicos, de los tratamientos didácticos de los temas de enseñanza y del aula de tecnología. En las siguientes secciones se describen estos aspectos de manera específica para cada modelo.

${ }^{3}$ Mediador: papel del profesor en el modelo pedagógico EFIT-EMAT, donde al incorporar los ambientes computacionales el docente es responsable de promover el intercambio de ideas y la discusión en grupo, al tiempo que actúa como intermediario entre el estudiante y la herramienta, es decir, en el ambiente computacional, al asistir a los estudiantes en su trabajo con las actividades de clase y compartiendo con ellos el mismo medio de expresión. 


\subsection{EL MODELO EMAT}

EMAT (enseñanza de las matemáticas con tecnología) es un modelo que contempla el uso de una variedad de piezas de tecnología (software especializado y calculadoras gráficas) estrechamente relacionadas cada una con las didácticas específicas de la geometría, el álgebra, la aritmética, la resolución de problemas y la modelación. En la mayoría de los casos, la construcción y el uso de estas piezas de tecnología cuentan con un sustento teórico y/o empírico, respectivamente, que respaldan su valor como herramientas mediadoras del aprendizaje en lo cognitivo y en lo epistemológico. En concreto, se incluye el uso de software de geometría dinámica para temas de geometría euclidiana; la hoja electrónica de cálculo para la enseñanza del álgebra, la resolución de problemas aritmético-algebraicos, y temas de probabilidad y de tratamiento de la información; la calculadora gráfica para la introducción a la sintaxis algebraica y a la resolución de problemas; el software para la simulación y la representación de fenómenos de movimiento para la enseñanza de la matemática de la variación y el cambio; y el software de modelación.

En un aula EMAT el maestro guía a los estudiantes en su trabajo con el ambiente computacional y con las hojas de actividades; interviene en este trabajo para garantizar que los alumnos alcancen cada vez mayores niveles de conceptualización; organiza discusiones colectivas, y es responsable de garantizar que la institucionalización del saber tenga lugar. En general, en el aula EMAT el maestro insta a los alumnos a:

- Explorar.

- Formular y validar hipótesis.

- Expresar y debatir ideas.

- Aprender comenzando con el análisis de sus propios errores.

Las actividades en el aula se organizan a partir de hojas de trabajo a través de las cuales los alumnos reflexionan sobre lo que han realizado con la computadora, y lo sintetizan para comunicarlo; por otro lado, las hojas de trabajo ya completadas proporcionan información al maestro acerca de la comprensión que los alumnos tienen de los conceptos matemáticos involucrados en la tarea. Con base en dicha información, el docente puede tomar decisiones sobre repaso y reforzamiento de temas, así como sobre nuevos diseños de actividades. 
Las hojas de trabajo también pueden ser utilizadas por el maestro como parte de la evaluación del aprovechamiento de los alumnos.

Éstos realizan las actividades en parejas frente a la computadora, de acuerdo con las hojas de trabajo. En tal modalidad se fomenta la discusión entre los estudiantes, quienes se ven en la necesidad de verbalizar y de expresar de múltiples formas sus razonamientos. En las discusiones colectivas que organiza el profesor se contrastan los diferentes acercamientos a una actividad determinada que se presentan en el grupo. Esos intercambios suelen tener un impacto significativo en los modos de apropiación del conocimiento.

La propuesta didáctica para utilizar dichas herramientas fue diseñada por expertos nacionales, tomando como base un modelo de aprendizaje colaborativo y un tratamiento fenomenológico de los conceptos.

\section{Herramientas de EMAT}

En EMAT se utiliza software abierto de contenido, donde el docente y el alumno deciden qué hacer con la herramienta, en lugar de que el propio programa de cómputo guíe de manera directa el trabajo del usuario, como ocurre con los llamados tutoriales. A continuación se describen brevemente las herramientas utilizadas en EMAT:

- Cabri-Géomètre: el software se rige por las reglas de la geometría euclidiana y permite a los alumnos explorar y elaborar conjeturas. La manipulación directa de representaciones formales de los objetos matemáticos ayuda a cerrar la brecha entre percepción y geometría, debido a que el software cuenta con elementos que brindan la posibilidad de animar las construcciones y de percibir transformaciones de trazos y figuras geométricas. Esto permite un acercamiento práctico a la enseñanza de la geometría.

- Hoja electrónica de cálculo: con esta herramienta se busca abordar dificultades bien conocidas en el aprendizaje del álgebra en la escuela secundaria, específicamente en la introducción de los alumnos a nociones fundamentales como las de función, variable, parámetro, fórmula, expresiones equivalentes y simbolización de patrones numéricos 0 geométricos. También se utiliza para enseñar a modelar y a 
resolver problemas aritmético-algebraicos, así como problemas planteados en el contexto de diferentes materias científicas.

- Calculadora Gráfica TI-92: es una herramienta de tecnología avanzada que contiene la mayoría de los paquetes de cómputo seleccionados para el modelo EMAT. Incluye las facilidades de cálculo de una calculadora científica con una capacidad gráfica. Incorpora recursos que la convierten en un medio para abordar distintos aspectos del currículo de matemáticas de la educación básica, tales como la manipulación numérica y algebraica, la graficación de funciones, el procesador geométrico Cabri-Géomètre, la edición y manipulación de matrices, y, en particular, tablas similares a una hoja de cálculo.

- SimCalc Math Worlds: con apoyo de una simulación, se ofrece al estudiante la oportunidad de aprender matemáticas con un enfoque gráfico, lo cual le permite familiarizarse con la lectura y con la interpretación de gráficas relacionadas con fenómenos de movimiento. Gracias a esta herramienta es posible una introducción temprana a temas de la matemática de la variación y el cambio; de ahí que se considere que este software da la oportunidad de explorar ideas matemáticas avanzadas que contribuyen al desarrollo del pensamiento complejo. Puede notarse que los temas que se abordan con este software rebasan los límites del currículo vigente en la educación secundaria.

- Stella: es un paquete de cómputo que permite expresar y probar ideas acerca del funcionamiento de sistemas dinámicos reales mediante la construcción de modelos matemáticos. Este enfoque de modelación implica trabajar con conceptos complejos de la matemática que pudieran resultar fuera del alcance de los alumnos de secundaria. Sin embargo, Stella proporciona un paso intermedio en la representación, la cual se hace por medio de un diagrama y no con un tratamiento simbólico a partir de ecuaciones matemáticas, lo que favorece su uso didáctico. La modelación no es un tema que aparezca en el currículo vigente, pero las actividades con Stella permiten a los alumnos acercarse a ideas importantes en matemáticas a través de un ambiente de modelación. 


\section{El aula EMAT}

Un aula EMAT se compone de 16 computadoras, considerando que los alumnos trabajan en parejas y que un grupo máximo de 30 estudiantes es atendido por un maestro, quien también dispone de una máquina. Es deseable que las computadoras estén conectadas en red local y con acceso a Internet, aunque esto último no es indispensable. EI complemento del equipo es un juego de 16 calculadoras TI-92, un ViewScreen, un proyector de acetatos y una impresora (ver figura 1 ).

\section{FIGURA 1}

\section{Aula EMAT. Escuela Secundaria «Manuel Álvarez», Colima, México}

La selección de herramientas, el diseño de actividades y la disposición de la tecnología en un aula EMAT no son incidentales, ya que están íntimamente relacionados con el modelo pedagógico en el cual las componentes epistemológica y tecnológica son indisociables.

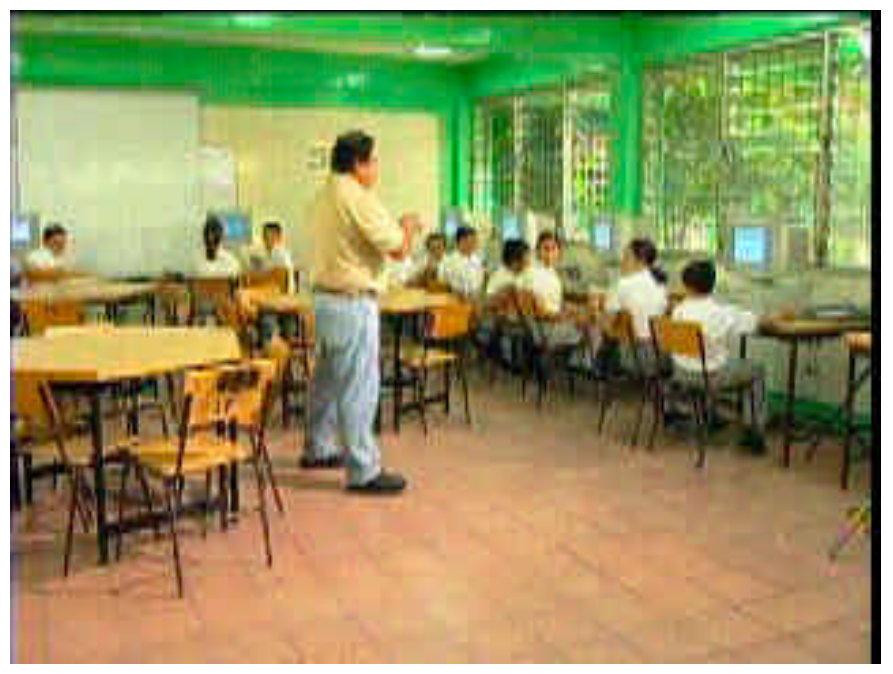

\subsection{EL MODELO EFIT}

EFIT (enseñanza de la Física con tecnología) es el resultado de la adaptación a la enseñanza de la Física en la escuela secundaria mexicana del modelo canadiense Technology Enhanced Science Secondary Instroduction, TESSI ${ }^{4}$. En 1997 el TESSI ya había sido probado por

4 Technology Enhanced Science Secondary Introduction/Introducción de Ciencias en Secundaria Mejorada con Tecnología. 
profesores durante más de cinco años en varias escuelas secundarias públicas de Canadá, localizadas en las inmediaciones de Vancouver.

A diferencia del modelo EMAT, en EFIT se incorporan al aula las computadoras y otros equipos (multimedia, sensores, interfaces, comunicación a distancia, correo electrónico, Internet, entre otros), en un proceso gradual: la primera fase utiliza la tecnología en sesiones expositoras por parte del maestro; en la segunda los estudiantes hacen uso de la tecnología con ayuda del maestro en un modelo de aprendizaje colaborativo (todos los alumnos realizan la misma actividad con la misma pieza de tecnología y en la propia sesión de clase), y, finalmente, en la tercera fase, de implementación total, los estudiantes hacen uso de la tecnología en forma independiente, guiados por el maestro en un modelo de aprendizaje cooperativo (diferentes grupos de alumnos trabajan en distintas estaciones de trabajo y con variadas piezas de tecnología, realizando actividades que son partes integrales de una tarea o proyecto colectivo amplio).

El maestro dedica el principio de la clase a explicar cómo se usa la tecnología, y proporciona las bases de las nociones de física involucradas en la actividad; a continuación, pone en práctica el modo colaborativo o el modo cooperativo para realizar dicha actividad, según se encuentre el grupo en la segunda o en la tercera fase de implementación del modelo EFIT.

Por medio de las guías de actividades, los estudiantes se acercan a la comprensión de los temas de física de cuatro maneras: realizando una simulación en la computadora; haciendo una actividad de laboratorio con el uso de sensores para recabar datos e ingresarlos a la computadora; llevando a cabo un experimento con un programa de cómputo o con el equipo de multimedia; y haciendo una prueba con equipo tradicional de laboratorio. Estos cuatro procedimientos dan lugar, de manera natural, a distintas estaciones de trabajo en una misma sesión de clase.

\section{Herramientas de EFIT}

- Interactive Physics: software de simulación de situaciones del mundo físico. Además de poder visualizar fenómenos que no pueden reproducirse en un laboratorio tradicional, el alumno puede comparar las simulaciones con el comporta- 
miento real de ciertos fenómenos. Es factible desplegar en pantalla una gran variedad de escenarios del mundo físico para ser estudiados.

- Sensores de Introducción a la Física Pasco: equipo consistente en sensores que se conectan a la computadora para medir diferentes magnitudes físicas (posición, velocidad, aceleración, fuerza, temperatura, sonido, voltaje, etc.). A diferencia del simulador, los sensores permiten realizar mediciones relativas a los propios fenómenos.

- NIH Image: software que posibilita la medición de cualquier imagen del macro y del micromundo en la computadora, la producción de imágenes de tercera dimensión, y la medición de imágenes de video digitalizadas.

- LXR Test: software de evaluación y autoevaluación que puede ser utilizado en cualquier asignatura. Esta herramienta permite hacer un banco de ítems de examen, creado y realimentado por los propios maestros para la elaboración de pruebas de opción múltiple o de respuestas abiertas.

- At Ease y ANAT: con este programa el maestro puede observar en la pantalla de su computadora el trabajo que se realiza en cada una de las computadoras de los alumnos. Así puede atenderlos individualmente en caso necesario. Con tal software también se puede controlar el acceso a los recursos de las computadoras y al sistema operativo desde un servidor.

- Netscape y Eudora: con Netscape los maestros y los estudiantes tienen acceso, vía Internet, a páginas con información y con programas de física de cualquier parte del mundo. Con Eudora pueden acceder a correos electrónicos que permiten la comunicación a distancia entre todos los participantes del proyecto.

\section{El aula EFIT}

En condiciones óptimas, un aula EFIT incluye 11 computadoras conectadas en red local, con acceso a Internet y con licencias de grupo de las piezas de software: de simulación, Interactive Physics (IP); de medición, NIH Image y Office; dos juegos de sensores, una impresora, una televisión y una videograbadora (ver figura 2). 
FIGURA 2

Aula EFIT. Escuela Secundaria Técnica n. ${ }^{\circ} 24$, Mérida, Yucatán

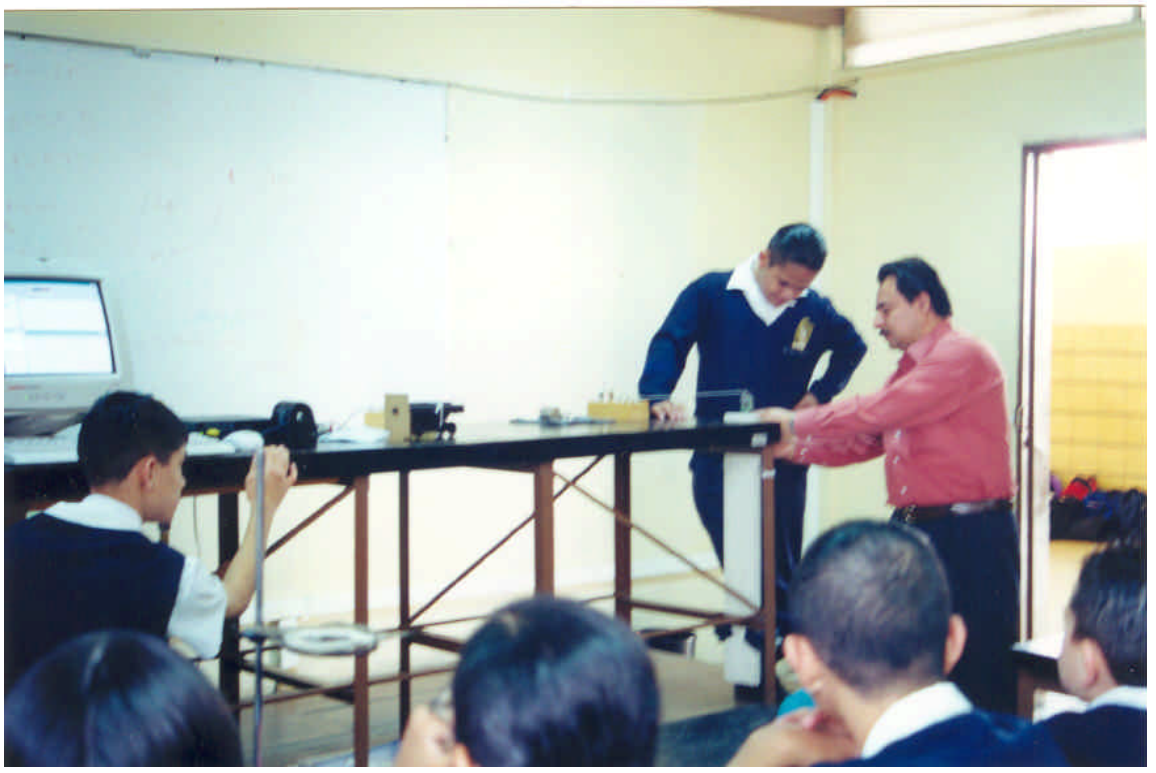

En el modelo EFIT confluyen el uso de nuevas tecnologías con los elementos de un laboratorio tradicional de física, de modo que tanto las guías de actividades como la disposición de la tecnología en el aula están determinadas por esta confluencia de herramientas y por un modelo pedagógico que transita de la clase expositora a una versión colaborativa, para culminar con una versión de aprendizaje cooperativo.

\section{PUESTA A PRUEBA DE LOS MODELOS EFIT Y EMAT}

A los modelos EFIT y EMAT antecede un buen número de experiencias en el uso de la informática educativa en proyectos de desarrollo en México, en la mayoría de los cuales se realizaron inversiones importantes en equipamiento de escuelas y en capacitación de recursos humanos. Sin embargo, no en todos los casos se documentó la experiencia, de modo que fue difícil juzgar el nivel de éxito o de logro de los objetivos planteados, y, por lo tanto, se careció de elementos para proponer su continuidad y/o su expansión. Una conclusión que se extrajo del análisis de antecedentes como estos consistió en la necesidad de validar modelos de uso de las TIC en educación en una etapa previa a su 
implementación a gran escala. Así, para EFIT y EMAT se ideó una fase piloto que abarcó un período de tres años (1997-2000), para la cual se estableció la infraestructura correspondiente en 28 escuelas secundarias, distribuidas en 14 entidades del país, dedicando 14 escuelas a cada uno de los modelos.

Los resultados de la fase piloto permitieron redefinir los modelos, de forma que resultase viable su aplicación generalizada. Hoy la expansión gradual de dichos modelos aparece como parte del Plan Nacional de Educación 2000-2006 de la SEP, y, en la práctica, al menos tres entidades han optado por su implementación inmediata en la totalidad de las escuelas secundarias (generales y técnicas) ${ }^{5}$. En esta sección se hace una breve descripción de los principales aspectos de la implementación y evaluación de la etapa piloto de EFIT y EMAT.

\subsection{Desarrollo curricular con tecnología}

En esta fase, a los criterios de selección de herramientas emanados de los principios básicos de los modelos (descritos en la sección 2) se adjunta el de adaptabilidad de tales herramientas a un desarrollo curricular con tecnología, apegado al currículo de matemáticas y física de la educación secundaria en México. A su vez, un requerimiento adicional para las propias herramientas es que permitan un tratamiento fenomenológico de los conceptos que quede plasmado en el diseño de las hojas de trabajo o en actividades que hayan de realizarse con ellas. Todo eso conduce a la elección del software abierto especializado, a las calculadoras gráficas y a los juegos de sensores descritos en la sección anterior.

Para la tarea de desarrollo curricular de EFIT y EMAT se conformaron grupos de expertos nacionales, asesorados por especialistas extranjeros, con experiencia tanto en las didácticas específicas de diferentes temas curriculares de matemáticas y física como en el desarrollo y uso de software de contenido especializado en dichos temas. Como resultado de este trabajo se elaboraron libros de actividades para

${ }^{5}$ En el sistema educativo mexicano existen tres tipos de escuelas secundarias: generales, técnicas y telesecundarias. Estas últimas, por lo general, se ubican en zonas de difícil acceso y hacen uso de la televisión educativa vía satélite. 
el alumno y guías de uso para el maestro, a fin de que cada uno de los modelos fuera publicado en versión revisada al término de la fase piloto por la SEP, el ILCE, el CONACYT y el CINVESTAV (SEP, 2000 y 2001) con los siguientes títulos:

- Matemáticas con la Hoja de Cálculo. EMAT.

- Geometría Dinámica. EMAT.

- Modelación. Matemática del cambio. EMAT.

- De los números al Álgebra en secundaria mediante el uso de la calculadora. EMAT.

- Guía para integrar los talleres de capacitación. EMAT.

- Enseñanza de la Física con Tecnología. EFIT.

Otro de los retos que hubo que enfrentar en esta producción de materiales didácticos fue el de conjugar un currículo elaborado sobre bases teóricas eminentemente constructivistas y un modelo pedagógico (con uso de tecnología) basado en una noción de aprendizaje colaborativo, más bien relacionada con las corrientes vygotskianas.

\subsection{EXPERTOS}

Como ya se señaló, el proceso de incorporación de los modelos EFIT y EMAT a las escuelas secundarias, con apego a los principios básicos, requirió la colaboración de expertos en didácticas específicas en física y matemáticas con amplia experiencia en el uso de entornos tecnológicos de aprendizaje. Aquí se hace referencia a expertos internacionales y nacionales, según se indica a continuación.

Los expertos internacionales son investigadores reconocidos en el ámbito educativo internacional, con amplia experiencia en el uso de nuevas tecnologías como apoyo a la enseñanza de matemáticas o ciencias en sus países, y por pertenecer a los grupos de investigación desarrolladores de los ambientes computacionales seleccionados para los modelos EFIT y EMAT. 
TABLA 1

Expertos internacionales

\begin{tabular}{|c|c|c|}
\hline Herramienta & Experto internacional & Institución \\
\hline Calculadora & Carolyn Kieran & $\begin{array}{c}\text { Universidad de Québec, } \\
\text { Canadá }\end{array}$ \\
\hline Hoja de cálculo & Rosamund Sutherland & $\begin{array}{c}\text { Universidad de Bristol, } \\
\text { Gran Bretaña }\end{array}$ \\
\hline Simcalc Math Worlds & Walter Stroup & $\begin{array}{c}\text { Universidad de Texas, } \\
\text { Austin,EE.UU. }\end{array}$ \\
\hline Stella & Ian Stevenson & $\begin{array}{c}\text { Universidad de Londres, } \\
\text { Gran Bretaña }\end{array}$ \\
\hline $\begin{array}{c}\text { Cabri Géomètre } \\
\text { Jean-Marie Laborde } \\
\text { seleccionadas para } \\
\text { EFIT. }\end{array}$ & $\begin{array}{c}\text { Universidad Joseph } \\
\text { Fourier, Grenoble, } \\
\text { canadiense expertos en } \\
\text { el modelo TESsI. }\end{array}$ & $\begin{array}{c}\text { Ministerio de Educación } \\
\text { y Open Learning } \\
\text { Agency, Canadá. }\end{array}$ \\
\hline $\begin{array}{c}\text { Evaluación global del } \\
\text { proyecto }\end{array}$ & Richard Lesh & $\begin{array}{c}\text { Universidad de } \\
\text { Massachussets, EE.UU. }\end{array}$ \\
\hline
\end{tabular}

La colaboración de los expertos internacionales en la puesta a prueba de los proyectos consistió en compartir sus conocimientos y experiencias en la elaboración y aplicación de un modelo pedagógico y didáctico acorde con las características del contexto nacional en un ambiente de cómputo particular, para el caso de EMAT, y, en el caso de EFIT, para la adaptación del modelo TESSI; en brindar asesoría y sugerencias académicas al experto nacional para orientar el diseño del material didáctico (actividades y hojas de trabajo) que debía ser utilizado por profesores y estudiantes en cada modelo; en proporcionar material que permitiera al experto nacional adquirir un conocimiento profundo de la herramienta correspondiente; y en realizar visitas de seguimiento a las escuelas participantes con el fin de proporcionar realimentación a los expertos nacionales sobre la puesta en marcha de los proyectos.

Los expertos nacionales son académicos o investigadores reconocidos de instituciones con amplia experiencia en investigación en el 
ámbito educativo de ciencias y matemáticas ${ }^{6}$. Fueron los responsables de diseñar y de adaptar las propuestas didácticas para cada modelo; de diseñar los materiales didácticos (actividades y hojas de trabajo) para profesores y alumnos, además de tener a su cargo el entrenamiento de instructores y de profesores. Mantuvieron un estrecho contacto con instructores y con maestros a fin de facilitar la implementación de cada una de las herramientas.

\subsection{SEDES EFIT-EMAT}

Tal como se dijo, se seleccionaron 14 escuelas por modelo, dos en cada entidad. Se optó por elegir los estados en los que ya se hubieran desarrollado proyectos de informática educativa y/o que contaran con instituciones de investigación educativa. Las sedes seleccionadas para probar los modelos se indican en el siguiente mapa.

FIGURA 3

\section{Sedes EFIT y EMAT}

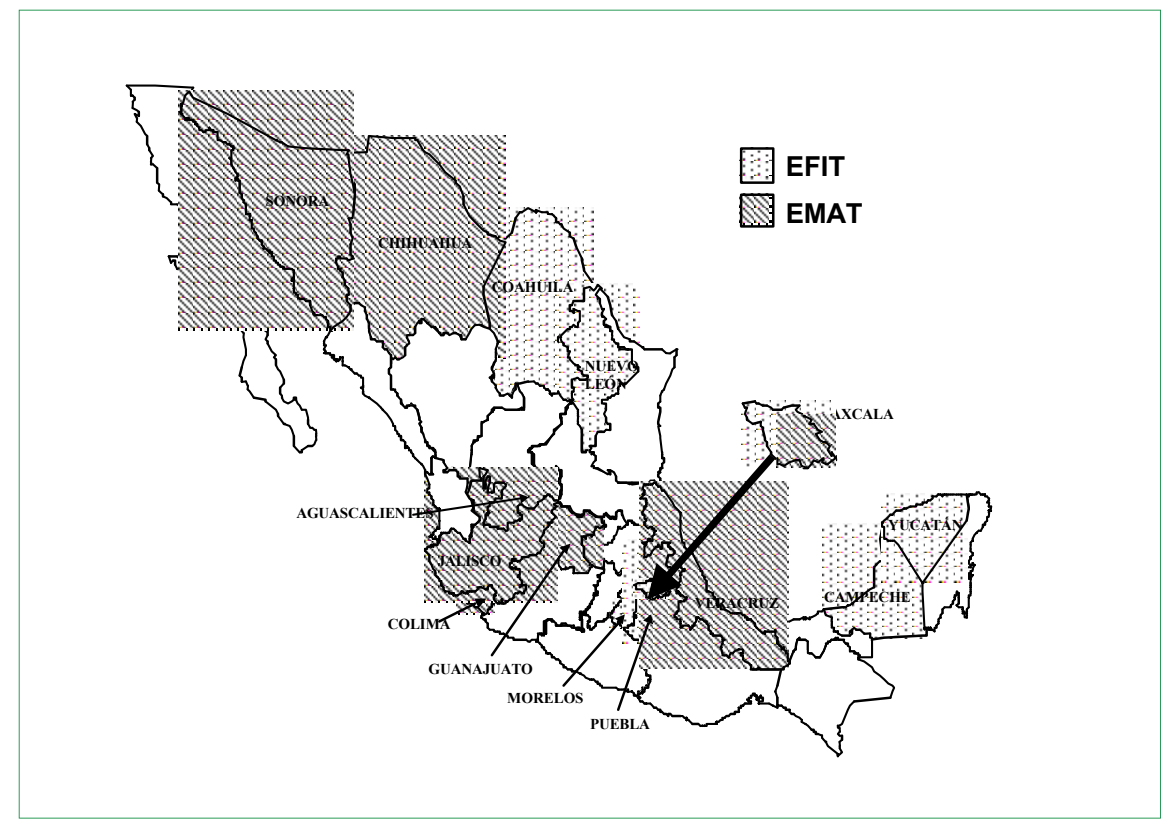

${ }^{6}$ Centro de Investigación y Estudios Avanzados del IPN (CINVESTAV), Instituto Tecnológico Autónomo de México (ITAM), Universidad Nacional Autónoma de México (UNAM), Universidad Pedagógica Nacional (UPN). 
En cada estado se designó una ciudad, donde fueron seleccionadas dos escuelas que se equiparon con la infraestructura necesaria (descrita en el apartado anterior), atendida cada una por un profesor responsable del proyecto y asesorado por un instructor.

\subsection{ESCUELAS EFIT-EMAT}

Las autoridades educativas de los estados participantes tuvieron la responsabilidad de escoger las dos escuelas donde se implementarían los modelos. No fue necesario que las designadas sobresalieran por alguna característica especial, pero sí que se comprometieran a participar en el proyecto durante un periodo mínimo de dos años; a otorgar el apoyo y las facilidades necesarias para el buen desarrollo de los proyectos; a designar para uso exclusivo del proyecto un salón de tamaño y mobiliario apropiados para albergar el equipo de EFIT y/o EMAT. En cada escuela participante se fijó el compromiso de remitir las clases de matemáticas o de física, según el caso, a las aulas EMAT o EFIT.

\subsection{INSTRUCTORES EFIT-EMAT}

Otro papel que se encomendó a las autoridades educativas locales, en cada una de las sedes, fue el de involucrar al personal interesado en participar en el proyecto, con el propósito de formarlo como experto en el modelo que había que poner a prueba para que se desempeñara como instructor de los profesores participantes. Por el tipo de actividad que tendría que cumplir para apoyar y orientar el trabajo de los profesores, este personal debía estar familiarizado con el currículo vigente de matemáticas y de ciencias de la educación secundaria, tener experiencia docente en este nivel educativo, y mostrar disposición al uso de nuevas tecnologías y a la aplicación de propuestas didácticas innovadoras.

Las principales funciones que debía desempeñar el instructor eran tener a su cargo la preparación de los profesores que pondrían en marcha el proyecto en las escuelas participantes; supervisar el trabajo de los docentes con el fin de procurar un desarrollo adecuado de las propuestas didácticas y pedagógicas diseñadas para cada modelo; mantener contacto permanente con los demás instructores, con los profesores participantes en su ciudad y con los expertos nacionales y extranjeros. 
Los instructores recibieron capacitación de los expertos nacionales y extranjeros sobre las herramientas y los modelos propuestos a través de talleres y de asesorías

\subsection{PROFESORES EFIT-EMAT}

Los docentes que participaron en el proyecto fueron designados por las autoridades locales, en colaboración con el instructor. Algunas de las características de los profesores seleccionados fueron: ser especialistas en matemáticas o física, tener amplia experiencia en impartir clases de ambas materias en secundaria, mostrar actitud innovadora y disposición al cambio, poseer capacidad para poner en práctica los materiales propuestos para cada proyecto, manifestar disposición para asistir a talleres de capacitación sobre el uso del modelo, comprometerse con el proyecto por un mínimo de dos años, tener -aunque no se consideró indispensable- cierta familiaridad en el manejo de computadoras, y disposición para explorar alternativas de enseñanza dentro del sistema educativo.

\subsection{CAPACITACIÓN}

Tal como se señaló, el equipo de especialistas en cada herramienta seleccionada para EFIT y EMAT fue responsable académico de brindar capacitación a instructores y profesores. Este grupo de expertos nacionales recibió asesoramiento e instrucción de expertos internacionales. Esto se ilustra en el siguiente esquema:

FIGURA 4

Esquema de capacitación

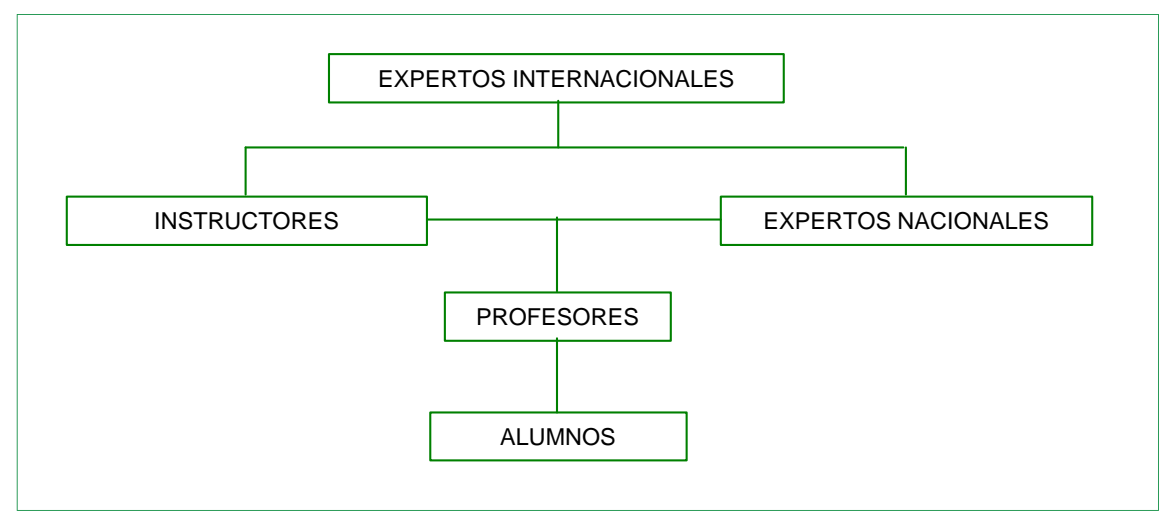


Para iniciar la fase piloto, los instructores y profesores recibieron capacitación en los aspectos técnicos y pedagógicos de cada modelo.

\subsection{IMPLEMENTACIÓN DE EMAT}

Por cada dos ciudades se trabajó al principio con un solo ambiente computacional, poniendo a prueba cuatro herramientas: CabriGéomètre, SimCalc, Hoja de Cálculo y Stella. Transversalmente a cada una de ellas, se utilizó la calculadora TI-92. Por las características propias de esta herramienta, fue factible su presencia generalizada a más corto plazo dentro del aula.

En el primer ciclo escolar (1997-1998) se pusieron a prueba la Hoja de Cálculo en primer grado, Cabri-Géomètre en segundo grado, Simcalc y Stella en tercero, y la calculadora gráfica en los tres. En los subsiguientes ciclos se realizaron las primeras acciones de expansión al incorporar más maestros en cada escuela, y al proporcionar la capacitación necesaria en una nueva herramienta. De esta manera, tras esos ciclos escolares los profesores recibieron capacitación en al menos tres de las herramientas.

Al finalizar los tres años durante los cuales se puso a prueba EMAT, el personal capacitado fue de 99 profesores, quienes atendieron a 10.526 estudiantes de las 14 escuelas secundarias participantes.

De las experiencias derivadas de la etapa piloto de EMAT se concluye que las herramientas que los maestros incorporaron con mayor facilidad a su práctica docente fueron la hoja electrónica de cálculo, la calculadora gráfica y el software de geometría dinámica. De ahí que para la fase de expansión del proyecto se propusiera la capacitación inicial de los maestros en la aplicación de estas tres herramientas.

Stella y SimCalc Math Worlds no se incluyeron en las acciones de expansión de EMAT, debido a que estas herramientas abordan contenidos que van más allá del currículo de matemáticas vigente, y a que representaron en su manejo un alto grado de dificultad para maestros y alumnos. Sin embargo, se prevé su futura incorporación a las prácticas en el aula, ya que con ellas se desarrollan ideas matemáticas como la modelación, la recursividad y la matemática del cambio, las cuales es muy probable que pasen a formar parte del repertorio de conocimientos y habilidades de los estudiantes de secundaria en la era digital. 


\subsection{IMPLEMENTACIÓN DE EFIT}

De acuerdo con el modelo TESSI, la incorporación de la tecnología en EFIT fue gradual, en tres fases, como ya se ha dicho. El modelo EFIT se puso a prueba en el primer ciclo escolar con estudiantes de segundo grado (Física I), y en el siguiente se incorporaron y capacitaron nuevos profesores para cubrir los contenidos del tercer grado (Física II).

Una vez concluida la fase experimental de EFIT, el personal capacitado fue de 58 profesores, quienes atendieron a 5.068 estudiantes de las 14 escuelas secundarias participantes.

Como resultado de la fase piloto de EFIT, se detectó la necesidad de hacer un ajuste en las piezas de tecnología propuestas por el modelo TESSI, reduciendo el modelo al uso de Interactive Physics y a un juego de sensores. Con estas herramientas se cubrió un gran número de contenidos del currículo de física para secundaria, lo que haría viable la implementación generalizada de EFIT en la escuela secundaria pública.

\section{EVALUACIÓN DE LA ETAPA PILOTO DE EFIT Y EMAT: EL PROYECTO DE INCORPORACIÓN DE LAS TIC A LA CULTURA ESCOLAR}

Por la magnitud de la inversión en equipo y por la formación de recursos humanos, que representarían una expansión a nivel nacional de Ios modelos EFIT y EMAT, y por las implicaciones en el aprendizaje de los alumnos y en las transformaciones de las prácticas en el aula, era clara la importancia de articular al proyecto piloto un proyecto de investigación (evaluación) cuyos resultados sirvieran de base para la toma de decisiones en las etapas posteriores a 1999. Así, en 1997 se elaboró la propuesta de investigación Incorporación de nuevas tecnologías a la cultura escolar: la enseñanza de las matemáticas y la física en la escuela secundaria ${ }^{7}$, que se sometió a concurso en la categoría de Proyecto de Grupo en la convocatoria de ese año del Consejo Nacional de Ciencia y Tecnología (CONACYT).

${ }^{7}$ Ref. n. ${ }^{\circ}$ G26338-S CONACYT, con sede en el CINVESTAV. Investigadores participantes: Teresa Rojano Ceballos, Eugenio Filloy Yagüe, Alfonso Martínez Vera, Simón Mochón Cohen, Luis Moreno Armella, Ana Isabel Sacristán Rock, Sonia Ursini Legovich y Gonzalo Zubieta Badillo (CINVESTAV); María Trigueros Gaisman (ITAM); Elisa Bonilla Rius (SEP); Juan Tonda Mazón (UNAM) y Tenoch Cedillo Ávalos (UPN). 
Con los recursos obtenidos del CONACYT se puso en marcha el proyecto de investigación por un periodo de cinco años, con el propósito de investigar:

- El papel que juegan diferentes piezas de tecnología en el desarrollo de habilidades y en el aprendizaje de contenidos curriculares específicos de matemáticas y de ciencias.

- El papel que juegan estas herramientas tecnológicas como mediadoras en el aprendizaje escolar de los estudiantes.

- De qué manera influyen la tecnología y el modelo de aprendizaje colaborativo en el aula en la transformación de la cultura científica y matemática escolar.

- De qué manera afecta al estudiante la presencia de las tecnologías de la comunicación y de la telepresencia para la incorporación de nueva información en sus estrategias de aprendizaje y en la generación de nuevo conocimiento.

- Las diferentes formas de asimilación de la tecnología a la cultura escolar en distintas regiones del país.

\subsection{LA METODOLOGÍA DE INVESTIGACIÓN}

Se utiliza un acercamiento telescópico que consiste en partir de un estudio a gran escala, el cual sirve como base para desarrollar criterios de selección de sujetos y de grupos de sujetos participantes en el proyecto para llevar a cabo un estudio de casos.

En el estudio a gran escala se aplicaron diagnósticos a toda la población de estudiantes participantes en el proyecto, cuyos resultados se analizaron cuantitativa y cualitativamente. A partir de este análisis se elaboraron los criterios de selección de sujetos para el estudio longitudinal de casos. Este último se efectuó en ocho escuelas participantes (cinco en EMAT y tres en EFIT), a través de entrevistas individuales a estudiantes y de cuestionarios aplicados a profesores, a autoridades escolares y a padres de familia.

La evaluación se realizó en dos niveles: global y local. El global centró su atención en la comprensión de lo que ocurre en el aula de matemáticas o de física en su condición de parte constitutiva de una 
comunidad más amplia, que, a su vez, constituye un sistema complejo que incluye dos tipos de entidades que resultan de gran relevancia para el desarrollo del proyecto y para su evaluación: los maestros, mediante quienes se evalúa el modelo de enseñanza; las autoridades escolares, por medio de las cuales se evalúa el proceso de asimilación del proyecto con tecnología por la organización escolar, con base en sus expectativas y en la valoración de la presencia de la tecnología en la escuela; y los padres de familia, a través de quienes se analiza la relación del uso de la tecnología en el aula con las expectativas sociales respecto a su rol en la formación escolar y al futuro laboral de los estudiantes. El nivel local se concentra en un estudio longitudinal de casos sobre el aprendizaje específico de los alumnos a lo largo de su experiencia con alguno de los modelos (EFIT O EMAT), y sobre la usabilidad de las herramientas tecnológicas utilizadas, en estrecha relación con el perfil de los usuarios de las mismas.

\subsection{RESULTADOS DE LA INVESTIGACIÓN ${ }^{8}$}

\section{Seguimiento local}

- Modelo emat: I) En el estudio a gran escala se observa un progreso significativo de la población estudiada en el lapso de un ciclo escolar, en cuanto al uso de un lenguaje simbólico más abstracto que el que se registra al inicio del estudio, en el que predominaban los lenguajes natural y numérico. Este progreso puede atribuirse al uso intensivo de los alumnos del código de la hoja de cálculo y de la calculadora gráfica, al utilizar estos medios para la modelación y resolución de problemas algebraicos de enunciado. II) A través del uso del entorno de geometría dinámica, los alumnos logran caracterizar figuras geométricas planas, afinar sus concepciones de perímetro y de área, generalizar propiedades del círculo, y elaborar conjeturas a partir de actividades de exploración. III) El trabajo con las actividades de modelación (software Stella y SimCalc) representan mayor dificultad para los alumnos que el trabajo en los otros entornos. Sin

8 Los resultados obtenidos con el trabajo de evaluación e investigación se encuentran documentados en más de 80 publicaciones, cuyas referencias pueden consultarse en <http://sippic.main.conacyt.mx:7777/indexSol.htm>. 
embargo, al final del ciclo escolar logran analizar fenómenos de movimiento en sus versiones gráfica y numérica.

Los resultados I a III surgieron de forma general en el estudio a gran escala, y se confirmaron y profundizaron en el estudio de casos.

- Modelo EFIT: IV) la comparación entre los resultados del ante y del post-cuestionario refleja un progreso de los estudiantes hacia el uso de un lenguaje más propio de las ciencias cuando se expresan en el aula. V) Las herramientas que resultaron de mayor utilidad para la conceptualización fueron el simulador Interactive Physics y los sensores de movimiento. Después de la fase experimental se procedió a mejorar aquellas actividades que mostraron deficiencias de diseño. VI) Tanto en el proyecto de Matemáticas como en el de Física, alumnos y maestros valoraron poder sistematizar el trabajo en clase con base en las hojas de trabajo o en las guías de actividades.

\section{Seguimiento global}

La asimilación por parte del maestro del modelo de uso de la tecnología para la enseñanza fue un proceso paulatino, pero al cabo de un ciclo escolar los docentes participantes descubrieron un modo de intercambio de ideas matemáticas o científicas con los alumnos a través de la tecnología y de las actividades diseñadas, se apercibieron del surgimiento de una variedad de estrategias de resolución de un mismo problema, e hicieron conscientes y explícitas sus propias deficiencias conceptuales en la enseñanza. Las autoridades escolares indicaron que el uso de la tecnología en la enseñanza tiene repercusiones en la organización escolar, y que la escuela ve incrementada su demanda de inscripción a partir de la presencia de la tecnología en el aula. Los padres de familia apoyaron el proyecto con su participación (ayudando a acondicionar el aula de tecnología), y mostraron interés en que sus hijos trabajaran con la computadora, aunque manifestaron inquietud acerca del cumplimiento del programa escolar porque no identificaban los temas curriculares con las actividades de EMAT y EFIT. 


\section{Extensión de la investigación}

En una fase posterior se pusieron a prueba materiales de sensibilización para la incorporación de nuevas generaciones de profesores al proyecto; los materiales se produjeron en versión CD-ROM, y se preparó su montaje en Internet para su uso a distancia. Se hicieron pruebas de aplicación de evaluaciones en línea para alumnos, lo que permitió utilizar este sistema de manera confiable en las fases de expansión del modelo. Se adaptó el modelo EMAT al sistema de Telesecundarias, y se llevó a cabo un estudio piloto en un grupo de escuelas de este sistema, obteniendo resultados similares a los de las fases anteriores. Se aplicaron cuestionarios para un estudio de género con uso de la tecnología; sus resultados sugirieron que el trabajo en entornos de tecnología tiene un efecto más acelerado en los niños que en las niñas, pero que a medio plazo (dos ciclos escolares) la tecnología tiende a homogeneizar el desempeño escolar en matemáticas en grupos de ambos sexos.

Los resultados de esta última fase ayudaron a elaborar el modelo de expansión en las siguientes direcciones: número de escuelas participantes, cobertura geográfica, cobertura curricular, variedad de herramientas, niveles escolares y subsistemas del sistema de educación básica (telesecundarias, centros de maestros y escuelas normales de maestros).

\section{CONCLUSIONES}

\subsection{EFIT-EMAT Y EL SISTEMA EDUCATIVO}

Disponer de un modelo probado y de los materiales educativos relacionados a partir del proyecto de grupo Incorporación del uso de las nuevas tecnologías a la cultura escolar, ha permitido incluir en el Plan Nacional de Educación (PNE) (SEP, 2001) metas concernientes al uso específico de las TIC en matemáticas y en ciencias en la educación básica para el período 2001-2006.

Esta concreción de la utilización de los resultados del mencionado proyecto da cuenta de sus implicaciones para el mejoramiento de la enseñanza en matemáticas y ciencias en el sistema de educación básica. En particular, se puede concluir que los estudiantes de educación 
secundaria serán a medio plazo beneficiarios directos de los logros alcanzados por los alumnos participantes en el plan piloto en cuanto a conceptualización y desarrollo de habilidades de exploración, a elaboración/ verificación de conjeturas, a resolución de problemas, a modelación de fenómenos del mundo físico, y a expresión en lenguaje matemático y científico en el aula. Otros beneficiarios serán los maestros de matemáticas y de ciencias de ese nivel, quienes participarán de los programas de capacitación contemplados en el modelo y en el PNE. Mediante tales programas, los maestros podrán transformar sus prácticas en el aula en un modelo que enfatice lo indispensable de su participación en los procesos de aprendizaje mediado por entornos de tecnología.

Los resultados de los cuestionarios y de las entrevistas aplicados a padres de alumnos participantes revelan aspectos culturales del papel que juega este grupo dentro de la escuela, en la implementación de programas de innovación educativa que involucran el uso de tecnología.

Finalmente, los efectos provenientes del estudio global 0 sistémico sugieren que no sólo es factible modificar las prácticas dentro del aula de matemáticas y de ciencias a partir del uso de las TIC, sino que se hace necesaria una reorganización escolar de conjunto, en la cual los directivos y los padres de familia participen en los procesos de aculturación que tienen lugar durante la asimilación del nuevo modelo educativo.

\subsection{EFIT-EMAT Y LA METODOLOGÍA DE SEGUIMIENTO Y EVALUACIÓN}

La posibilidad de haber realizado un estudio Iongitudinal estricto (seguimiento de tres generaciones de alumnos y de maestros participantes a lo largo de tres ciclos escolares consecutivos), permite concluir, a partir de los resultados obtenidos, que el proceso de asimilación del uso de las TIC en la enseñanza es parsimonioso, es decir, que en el caso de este proyecto la evidencia de éxito con el nuevo modelo en grupos núcleo dentro de la escuela ha generado una demanda natural de expansión del uso del modelo hacia otros grupos de alumnos, así como hacia escuelas vecinas (Ios casos de Colima y Aguascalientes ilustran este hecho), y hacia entidades cercanas (casos de Coahuila, Durango y San Luis Potosí). Este proceso de contagio es lento y gradual, lo que tiene implicaciones para la planeación de medio y largo plazo de la incorporación del uso de las TIC de manera masiva en el sistema escolar. 
Por su parte, el acercamiento telescópico en la observación y seguimiento del desarrollo del proyecto permitió profundizar el análisis de los resultados obtenidos en el estudio a gran escala, a partir del estudio longitudinal de casos, el cual se enfocó a analizar tanto la evolución de los alumnos en la conceptualización y desarrollo de habilidades, como los procesos cognitivos que tienen lugar en dicha evolución. De este modo, las explicaciones de lo que se detecta a nivel macro con los datos y procesamientos estadísticos, se amplían con los resultados del tratamiento cualitativo (nivel micro).

\subsection{EFIT-EMAT Y LA INVESTIGACIÓN CIENTÍFICA BÁSICA}

Los resultados del estudio longitudinal de casos también confirman los obtenidos por estudios previos sobre el uso de herramientas específicas de TIC en la enseñanza de matemáticas y de ciencias realizados en condiciones de laboratorio o en desarrollos a pequeña escala. De este modo, se muestra la relevancia de utilizar resultados de investigación básica en el diseño e implementación de programas de desarrollo e innovación en un sistema educativo.

\subsection{EVALUACIÓN DE LA EVALUACIÓN}

Cabe mencionar que, en una etapa posterior a la conclusión del proyecto Incorporación de las TIC a la Cultura Escolar, el CONACYT solicitó a la agencia Irwin-Invertec la evaluación externa de una serie de proyectos de investigación, financiados por el propio Consejo (entre ellos el mencionado proyecto), a fin de analizar el impacto social y la relevancia científico-tecnológica de los resultados de dichas investigaciones aplicadas.

Los resultados de la evaluación realizada por Irwin-Invertec del proyecto mencionado están consignados en el informe de caso Aplicación de las Tecnologías de la información a las matemáticas y las ciencias en las escuelas secundarias (CONACYT, 2002). En términos generales este informe confirma, a través de su propio levantamiento de datos, algunos de los resultados conseguidos por el proyecto, que textualmente dice: «los resultados a nivel de piloto muestran un alto porcentaje (75\%) de estudiantes con un historial previo de fracaso en matemáticas que han logrado acreditar el curso con resultados que no difieren mucho de los 
alcanzados por los estudiantes más avanzados. Esto, sumado al uso del lenguaje matemático y científico, conciencia en los maestros del nivel de conocimiento en la materia enseñada, transformación total de las prácticas escolares en matemáticas y ciencias y valoración por parte de los padres, y muestra la efectividad que ha tenido a la fecha el nuevo modelo didáctico-pedagógico» (p. 86).

Pero también en el informe se hacen señalamientos en la dirección de las limitantes del proyecto para el avance del programa; entre ellos se hace referencia a ciertas deficiencias detectadas en la formación de los docentes, no sólo en el manejo de tecnología sino también en conceptos asociados a la materia que enseñan. «Se adiciona a esto la gran diferencia entre el enfoque didáctico tradicional con el nuevo, que impone exigencias adicionales a estos profesionales. Estas deficiencias deben ser tomadas en consideración para re-diseñar el programa de formación de maestros [...]» (p. 86).

Otra de las limitantes señaladas es la gran resistencia al cambio presente en un conjunto de maestros que no ha tenido contacto directo con la informática educativa.

Las limitantes arriba señaladas, que igualmente habían sido registradas por el proyecto de investigación evaluado, advierten de la necesidad de una reformulación o ampliación de las premisas del proyecto educativo EFIT-EMAT, en el sentido de incorporar el papel del maestro con mayor énfasis del que aparece en el planteamiento inicial, y de dar mayor importancia en el proceso de capacitación de los docentes a la revisión de los conceptos fundamentales de las materias de enseñanza.

\section{NOTA FINAL}

La idea de escribir un artículo monográfico del proyecto EFITEMAT y del correspondiente proyecto de investigación, tiene la finalidad de diseminar los resultados de una experiencia con uso de las TIC, que, si bien fue desarrollada en un sistema educativo particular, de ella pueden extraerse lecciones aprovechables por otros sistemas; por ejemplo, en los niveles metodológico, logístico y de definición de políticas educativas en el uso de los nuevos entornos tecnológicos de aprendizaje. 


\section{BIBLIOGRAFÍA}

BOERS-VAN OOSTERUM, M. (1990): Understanding of Variables and Their uses Acquired by Students in Traditional and Computer-Intensive Algebra, Ph. D. diss. University of Maryland College Park. Londres, Routledge.

CRook, C. (1994): Computers and the Collaborative Experience of Learning,

CONACYT (2002): Informe del caso: aplicación de las tecnologías e información a las matemáticas y las ciencias en la enseñanza en las escuelas secundarias, IrwinInvertec.

DUNHAM, P. y Dick, T. (1994): «Research on Graphing Calculators», Mathematics Teacher, 87(6), Lugar Editorial, pp. 440-445.

FACER, K; Furlong, J; SUTHERLAND, R. y FURLONG, R. (2000): «Home is Where the Hardware is: Young People, the Domestic Environment and 'Access' to new Technologies», en Hutchby, I. y Moran-Ellis, J. (eds.): Childrens, Technology and Culture, Londres, Falmer Press.

LAVE, J. (1988): Cognition in Practice, Cambridge University Press, Cambridge. Madrid, Santillana.

MCFARLANE, A. (2001): El aprendizaje y las tecnologías de la información,

MCFARLANE, A.; BONNETT, M. y WiLLIAMS, J. (2000): «Assessment and Multimedia Authoring - A Technology for Externalising Understanding and Recognising Achievment», Journal of Computer Assisted Learning, 16, pp. 201-212.

Rogoff, B. y LAVE, J. (1984): Everyday Cognition: Its Development in Social Context, Harvard University Press.

Rojano, T. (1996): «Developing Algebraic Aspects of Problem Solving Within a Spreadsheet Environment», en Bednarz, N.; Lee, L. y Kieran, C. (eds.) Approaches to Algebra: Perspectives for Research and Teaching, Londres, Boston, Kluwer Academic Publishers.

WERTSCH, J. (1991): Voices of the Mind: A Sociocultural Approach to Mediated Action, Harvester Wheatsheaf.

\section{DIRECCIONES VIRTUALES}

- Calculadoras TI-92: <http://education.ti.com/us/product/graphing.html>.

- Cabri Géomètre: <http://www-cabri.imag.fr/>.

- Interactive Physics: <http://www.interactivephysics.com>.

- LXR Test:<http://www.|xrtest.com/>.

- NIH Image: <http://rsb.info.nih.gov/nih-image/index.html>. 
- Proyecto CONACYT: <http://sippic.main.conacyt.mx:7777/indexSol.htm>.

- Sensores Pasco:<http://www.pasco.com/>.

- SimCalc Mathworlds:<http://www.simcalc.umassd.edu/simcalcframe.html>.

- Stella:<http://www.hps-inc.com/>. 


\title{
Contactar
}

Revista lberoamericana de Educación

\author{
Principal OEI
}

